\title{
Development of a high-resolution mass spectrometry method for DNA adductomics investigation in colon cancer
}

\author{
Giorgia La Barbera ${ }^{\mathrm{a}}$, Katrine Dalmo Nommesen ${ }^{\mathrm{a}}$, Lars Ove Dragsted*a. \\ ${ }^{a}$ Department of Nutrition Exercise and Sports, University of Copenhagen, Frederiksberg, DK-1985
}

\section{-ABSTRACT}

Colorectal cancer (CRC) may develop after covalent modification of DNA by exogenous or endogenous genotoxic molecules coming from smoke, meat intake, inflammation or other factors. Several genotoxicants are known to cause DNA damage, but there is little evidence on their identity. DNA adductomics is a new research field, aiming to screen unknown DNA adducts by high resolution mass spectrometry (HRMS). However, due to the low abundance of DNA adducts, DNA adductomics presents several analytical challenges.

In this work, a sensitive untargeted DNA adductomics method was developed by using ultra-high performance liquid chromatography (UHPLC) coupled via electrospray ionization source (ESI) to quadrupole-time of flight MS (Vion-qTOF). Mobile phases with ammonium bicarbonate gave the best signal enhancement. The MS capillary voltage, the cone voltage and the detector voltage mainly affected the response of the DNA adducts. A low adsorption vial was selected for reducing analyte loss. A hybrid surface coated HSST3 premier column showed a minimal adsorption of the DNA adducts. The optimized method was applied to analyse DNA from calf thymus and cat colon by performing a MSE acquisition screening for the loss of - $\mathrm{dR}$, both in source and in the fragmentation spectra, and for the nucleobase fragment ions, to select the potential DNA adducts. Thirteen DNA adducts were observed in DNA from calf thymus and cat colon, showing good promise for the application of this untargeted method in future human studies. 


\section{- INTRODUCTION}

In the past few years, a new -omics science exploring the modifications of DNA from endogenous or exogenous genotoxicants has developed, DNA adductomics. ${ }^{1}$ The exposure of human DNA to genotoxic chemicals induces the formation of covalent DNA-adducts which, if not repaired, can lead to gene mutations, ultimately increasing the risk of cancer. ${ }^{2}$ The measurement of DNA-adducts is of fundamental importance in assessing the potential carcinogenic effects of the different exposures coming from diet and environment, and in understanding their mechanisms of action. ${ }^{1}$

Several analytical methods, such as immunochemical methods, ${ }^{32} \mathrm{P}$-postlabeling techniques and liquid chromatography coupled to mass spectrometry (LCMS), have been used for DNA adduct analysis over the past 30 years, the last being lately considered the gold standard technique. However, the limitations of the old MS instruments in terms of both sensitivity and selectivity only allowed to monitor a few targeted DNA-adducts at a time, not providing a global picture of the "DNA-adductome". ${ }^{3}$ The new technological advances of the last decades have led to the development of high resolution mass spectrometry (HRMS), which provides the measurement of accurate compound mass, allowing their identification with high confidence. HRMS, together with ultra-high performance (UHP)LC, have opened new horizons in the screening of unknown DNA-adducts and led to the development of this new research field. ${ }^{4}$

As with any new analytical science, DNA adductomics presents new challenges demanding new sample preparation protocols, chromatographic methods, data acquisition and data analysis approaches. The major analytical challenge in DNA adductomics is still the need for high sensitivity and selectivity due to the fact that DNA-adducts are present at trace levels and in very complex matrices. ${ }^{3,4}$ The hardest step in DNA adductomics is DNA-adduct identification. Whereas the traditional -omics sciences rely on software support, DNA adductomics is in a developmental phase. ${ }^{5,6}$ Some studies have been published with the aim of building a DNA-adduct database, but they generally focus on a limited class of exposures or DNA adducts. $^{7-10}$

In this present work, particular interest has been posed in the investigation of DNA adducts related to colorectal cancer (CRC). The analysis of CRC-related DNA adducts has been already carried out in the past with older techniques, showing promising results on the relationship between CRC carcinogenesis and specific genotoxicants coming from red meat intake, alcohol intake and smoking. ${ }^{11}$ However, the associations of CRC with these exposures have only been partially elucidated, ${ }^{12}$ requiring the development of a new and advanced method for profiling of the colon epithelial DNA adductome.

A sensitive UHPLC-HRMS based method was therefore developed for this purpose. An UHPLC coupled via an electrospray ionization source (ESI) to a quadrupole-time of flight MS (Vion-qTOF) was used for the optimization of the chromatographic and mass spectrometric method on a mixture of DNA adduct reference standards, in order to increase the sensitivity of the instrumental analysis. The developed method was used for the analysis of DNA adducts in DNA from calf thymus and from cat colon.

\section{- EXPERIMENTAL SECTION}

\section{Chemicals and materials}

For the UHPLC analysis milli-Q ultra pure water, methanol optima LC/MS grade (Thermo Fisher Scientific, Waltham, MA) and formic acid, acetic acid, ammonium acetate, ammonium formate, ammonium bicarbonate from Merck (St. Louis, MO.) were used. The following DNA adducts and nucleosides or nucleobases reference standards were purchased from Toronto Research Chemicals: 2'-deoxyadenosine monohydrate(dA); 2'-deoxyguanosine monohydrate (dG); thymidine (dT); 2'-deoxyuridine (dU); 2'-deoxyN6-methyladenosine (N6-Me-dA); 5-methyl-2'deoxycytidine (5-Me-dC); O6-methyl-2'deoxyguanosine(O6-Me-dG); 2'-deoxy-N3methyluridine(N3-Me-dU); N3-methylthymidine (N3Me-dT); N4,5-dimethyldeoxycytidine(N4,5-DiMedC); N2-ethyl-2'-deoxyguanosine (N2-ethyl-dG); N6(2-hydroxyethyl)-2'-deoxyadenosine (N6-2-OH-ethyldA); 8-oxo-2'deoxyguanosine (8-oxo-dG); etheno-2'deoxy- $\beta$-D-adenosine (1,N6- $\varepsilon$-dA); 3,N4-etheno- 2 'deoxycytidine (3,N4- $\varepsilon$-dC); 3-(2-deoxy- $\beta$-D-erythropentofuranosyl)pyrimido[1,2-a]purin-10(3H)-one (M1-dG); 3-(2-Deoxy- $\beta$-D-erythro-pentofuranosyl)3,5-dihydropyrimido[1,2-a]purine-6,10-dione (6-OxoM1-dG); $\gamma$-Hydroxy-1,N2-propano-2'-deoxyguanosine $(\gamma-\mathrm{OH}-1, \mathrm{~N} 2-\mathrm{PdG}) \quad(\gamma-\mathrm{OH}-\mathrm{Acr}-\mathrm{dG}) ; \quad \alpha-$ Methyl- $\gamma-$ hydroxy-1,N2-propano-2'-deoxyguanosine (mixture of diastereomers) ( $\alpha-\mathrm{Me}-\gamma-\mathrm{OH}-1, \mathrm{~N} 2-\mathrm{PdG})(\mathrm{Cro}-\mathrm{dG}) ; \mathrm{N}-$ (2'-deoxyguanosin-8-yl)-4-aminobiphenyl (C8-ABP- 
$\mathrm{dG}$ ); and N2-(deoxyguanosin-8-yl)-2-amino-3,8dimethylimidazo[4,5-f] quinoxaline (C8-MeIQx-dG). The following nucleosides reference standards were purchased from Merck: adenosine, cytidine (dC), uridine. Stock solutions of the DNA adduct standards were dissolved at 1 or $0.5 \mathrm{mg} \mathrm{mL}^{-1}$ in $\mathrm{MeOH}$ or a mixture of water and methanol. A stock solution was prepared containing all the standards at $20 \mu \mathrm{g} \mathrm{mL}{ }^{-1}$. The working solutions were diluted with water to concentrations ranging from $100 \mathrm{ng} \mathrm{ml}^{-1}$ to $1 \mathrm{ng} \mathrm{ml}^{-1}$. The following products for DNA extraction and hydrolysis were purchased from Merck: Ribonuclease A from bovine pancreas for molecular biology; Proteinase K from Tritirachium album BioUltra, for molecular biology; Sodium dodecyl sulfate (SDS) BioUltra, for molecular biology; Phenol : chloroform : isoamyl alcohol mixture $(25: 24: 1, v / v / v)$ BioUltra, for molecular biology; Deoxyribonucleic acid sodium salt from calf thymus, Type I; Deoxyribonuclease I from bovine pancreas, Type IV (DNaseI); Phosphodiesterase I from Crotalus atrox (Western Diamondback Rattlesnake), Type IV (PDEI); Alkaline Phosphatase from bovine intestinal mucosa (AP); Tris(hydroxymethyl)aminomethane hydrochloride (Tris $\mathrm{HCl}$ ); ethylenediaminetetraacetic acid (EDTA); sodium chloride $(\mathrm{NaCl})$; and magnesium chloride hexahydrate $\mathrm{MgCl}_{2} 6 \mathrm{H}_{2} \mathrm{O}$. Ethanol was from Thermo Fisher Scientific (Waltham, MA).

\section{DNA from calf thimus experiment}

DNA from calf thymus was hydrolyzed before analysis. In brief, $0.5 \mathrm{mg}$ of DNA were dissolved in 1 $\mathrm{mL}$ of incubation buffer $(10 \mathrm{mM}$ Tris- $\mathrm{HCl}$ and $5 \mathrm{mM}$ $\mathrm{MgCl}_{2}$, adjusted at $\mathrm{pH} 7$ ). Five hundred units of DNaseI were added and the sample was incubated overnight in a mixer at $37{ }^{\circ} \mathrm{C}$. The next day, additional 500 units of DNaseI were added to the sample together with 0.01 units of PDEI and 100 units of AP. The sample was incubated overnight in a mixer at $37^{\circ} \mathrm{C}$. The next day, two volumes of cold methanol were added to the sample for precipitating the proteins. The supernatant was collected and evaporated. The sample was dissolved with a total of $300 \mu \mathrm{L}$ of $\mathrm{H}_{2} \mathrm{O}: \mathrm{MeOH}$ (90:10, $v / v)$.

\section{DNA from cat colon}

Colon epithelial tissue was scraped off the resected colon obtained from a euthanized cat. The cat was euthanized at the University Hospital for Family Pets in Copenhagen following the National guidelines for ethics in animal experiments. Five hundred $\mathrm{mg}$ of epithelial tissue were grinded in liquid nitrogen. The sample was dissolved in $2.5 \mathrm{~mL}$ of the DNA digestion buffer (50 mM TRIS HCl, $10 \mathrm{mM}$ EDTA and $100 \mathrm{mM}$ $\mathrm{NaCl}$, adjusted at $\mathrm{pH} 8$ ). SDS $300 \mu \mathrm{L}, 10 \%$, and 250 units of proteinase $\mathrm{K}$ were added to the sample and incubated over night at $37^{\circ} \mathrm{C}$. The next day, 250 units of RNase were added and incubated again for two hours at $37^{\circ} \mathrm{C}$. DNA was extracted from the sample with 1 volume of phenol:chloroform:isoamil alcohol (25:24:1, $v / v / v)$ and mixed by inversion. After centrifugation at $1600 \mathrm{~g}$ for $10 \mathrm{~min}$, the upper phase was collected. Two volumes of cold $\mathrm{EtOH}$ were added, and the vial was inverted for DNA precipitation. After centrifugation at $1600 \mathrm{~g}$ for $10 \mathrm{~min}$, the supernatant was discarded and the DNA was washed with $3 \mathrm{~mL}$ of $\mathrm{EtOH}: \mathrm{H}_{2} \mathrm{O}$ (70:30, $v / v)$. The sample was inverted again followed by centrifugation at $1600 \mathrm{~g}$ for $5 \mathrm{~min}$. The supernatant was discarded, and the sample was air dried at room temperature for 10-15 min. The DNA was dissolved in $10 \mathrm{mM}$ Tris- $\mathrm{HCl}, 5 \mathrm{mM} \mathrm{MgCl} 2$, adjusted to $\mathrm{pH} 7$ and left overnight at $4{ }^{\circ} \mathrm{C}$. The day after, the DNA extraction yield $(0.89 \mathrm{mg})$ and purity $\left(\mathrm{OD}_{260 / 280}=1.87\right.$ $\mathrm{OD}_{260 / 230}=2.12$ ) were assessed by UV. The equivalent of $0.5 \mathrm{mg}$ of DNA was used for the further DNA hydrolysis procedure, as described for DNA from calf thymus.

\section{UHPLC-HRMS method optimization}

The analysis was performed on an $\mathrm{H}$ class Acquity UHPLC coupled to a Vion-IMS-qTOF (Waters, Milford, MA) via a heated electrospray ionization (ESI) source. The UHPLC system was equipped with a quaternary pump and an autosampler thermostated at $10{ }^{\circ} \mathrm{C}$. A C18 HSS T3 column $(100 \times 2.1 \mathrm{~mm}, 1.8 \mu \mathrm{m}$ particle size) (Waters) was used at $0.4 \mathrm{~mL} \mathrm{~min}^{-1}$ and at $50{ }^{\circ} \mathrm{C}$.

Different mobile phases were compared in order to improve chromatographic separation and sensitivity, and to decrease in source fragmentation and adduct formation. Details on the mobile phase comparison experiment are provided in the Supporting Information (Table S1). The best chromatographic condition used $10 \mathrm{mM} \mathrm{NH}_{4} \mathrm{HCO}_{3}$ as mobile phase $\mathrm{A}$ and $\mathrm{MeOH}$ with $10 \mathrm{mM} \mathrm{NH}_{4} \mathrm{HCO}_{3}$ as mobile phase B.

Several MS spectrometric parameters and acquisitions were evaluated for increasing the sensitivity and decreasing the in-source fragmentation of DNA adducts. Different values of capillary voltage, sampling cone voltage, source temperature, desolvation 
temperature, desolvation gas, collision energy, mass range, profile or automatic mode in the quadrupole isolation, and detector voltage were tested as summarized in the Supporting Information (Table S2). The optimal tuning parameters of the Vion-IMS-qTOF were: capillary voltage $0.5 \mathrm{kV}$; sampling cone voltage $30 \mathrm{~V}$; source temperature $110{ }^{\circ} \mathrm{C}$; desolvation temperature $600{ }^{\circ} \mathrm{C}$; desolvation gas $800(\mathrm{~L} / \mathrm{h})$; collision energy $6 \mathrm{eV}$; cone gas $50(\mathrm{~L} / \mathrm{h})$. The detector voltage was set to $3000 \mathrm{~V}$. The Vion-IMS-Q-TOF was operated in $\mathrm{MS}^{\mathrm{E}}$ acquisition mode and samples were acquired in positive polarity mode. For both the low and high energy trace the scan range was $50-1000 \mathrm{~m} / \mathrm{z}$ and the scan time $0.4 \mathrm{~s}$. For the high energy trace optimization of the collision energy was performed to obtain an informative fragmentation pattern, finally choosing a mass energy ramp ranging from 20 to 50 $\mathrm{eV}$.

Finally, in order to reduce eventual adsorption processes of DNA adducts, low adsorption injection vials and a low adsorption column were tested by analysing the mix of DNA adduct reference standards at concentration between 1 and $10 \mathrm{ng} \mathrm{ml}^{-1}$. In particular, five different injection vials, i.e. LC-MS certified clear glass Vial, TruView Vial and Quan Recovery Max Peak (all Waters), Low Adsorption Vial (Supelco, Merck, St. Louis, MO) and Reduced Surface Activity RSA-Pro Vial (Microsolv, Greater Wilmington, NC) were compared by 14 repeated injections over a period of $24 \mathrm{hr}$. The vial giving the least adsorption and the highest stability of the signal over time, i.e. the Low Adsorption Vial, was chosen for the following column comparison. The HSS T3 column used in the previous experiments was compared with a hybrid surface coated Premier C18 HSS T3 column $(100 \times 2.1 \mathrm{~mm}, 1.8 \mu \mathrm{m}$ particle size) (Waters); the latter showed better results and was then used for analysis of real samples.

The final optimized conditions were used for the analysis of the hydrolyzed DNA from calf thymus and from cat colon, using the following gradient: 0-1 min (5\% B), 1-21 $\min (0-99 \% \mathrm{~B})$, followed by a $2 \mathrm{~min}$ wash at $99 \% \mathrm{~B}$ and 2 min equilibration at $5 \% \mathrm{~B}$.

For performing all the mentioned analysis the mass spectrometer was periodically cleaned and externally calibrated every 2 weeks using the calibration solutions Major Mix (Waters). Lock mass correction was continuously applied during the runs by injecting $15 \mu \mathrm{L}$ $\min ^{-1}$ of leucine encephalin solution (Waters) every 5 min. Three technical replicates were performed for each condition evaluated. For the evaluation of the chromatographic conditions in particular, the three replicates were run after flowing the mobile phase for $1 \mathrm{hr}$ and running two blanks for assuring column conditioning. Also, samples were run continuously day and night to minimize instrumental variability. To evaluate the mass spectrometric conditions and for vial comparisons, continuous injections over $4 \mathrm{hrs}$ of a DNA adduct mix at high concentration were performed before the analysis in order to avoid any column passivation effect. For the comparison of the two columns, continuous injections over $4 \mathrm{hrs}$ of a blank sample were performed before the analysis in order to test the passivation effect on the two columns. For all the analysis the injection volume was $5 \mu \mathrm{L}$.

\section{Data analysis and DNA adduct identification}

Raw data files obtained for the optimization of the chromatographic and mass spectrometric parameters were acquired by UNIFI software (version 1.9.4.053) (Waters) and transformed into .mzml format by using the MSconvert tool (http://proteowizard.sourceforge.net/tools.shtml). ${ }^{13}$

The converted files were then imported into MZmine (http://mzmine.github.io) for further analysis. ${ }^{14}$ Several values such as peak area, full width at half maximum (FWHM) and the asymmetry factor, were extrapolated from the analyzed chromatographic runs. Briefly, the Targeted Peak Detection module was used for integrating the peaks related to the adducts and in source fragments of the DNA adduct standards. Peak integration was checked and manually corrected when needed. The RANSAC alignment was used for aligning the chromatographic runs acquired under the same chromatographic conditions, whereas the Join Aligner was used for aligning the runs acquired with different chromatographic conditions, since the retention time could be neglected in this kind of alignment. The parameters used for the data analysis with MZmine are reported in the Supporting Information (Table S3.1). Retention time, peak area of every single adduct as well as the ratio of $[\mathrm{M}+\mathrm{H}]^{+}$over any other adduct or in source fragment, full width at half maximum (FWHM), asymmetry factor, peak capacity, and resolution were extrapolated as reported in the paper of La Barbera et al. ${ }^{15}$ and used for the comparison. Further details on the calculations are reported in the Supporting Information S3.

Raw data files related to the analysis of DNA from calf thymus and cat colon were acquired and analyzed by 
UNIFI software (version 1.9.4.053) (Waters) in four steps. The first step consists in peak picking and alignment of the chromatographic runs. The second step consists in the search for possible adducts. These were chosen based on the typical ionization behavior of the DNA adduct reference standards, in the low energy trace. Among the adducts also $[\mathrm{M}-\mathrm{dR}+\mathrm{H}]^{+}$was included, even if it is formally an in source fragment due to the loss of deoxyribose (-dR). The third step consists in the search for the neutral loss of $-\mathrm{dR}$ between the low and the high energy trace. The fourth step consists in the search for common fragments, i.e. the ion masses related to the unmodified nucleobases, adenine, cytosine, guanine, thymine, and uracil in the high energy trace. The parameters used for the data analysis with UNIFI are reported in the Supporting Information (Table S3.2). A manual investigation of the fragmentation spectra of the selected compounds was performed by running a targeted MS/MS acquisition at a collision energy consisting in a ramp of 20-50 eV. Identification confidence levels were associated with the DNA adducts based on the work of Schymanski et al. ${ }^{16}$ but with some modifications: level 1) for compounds identified by reference standard retention time and MS/MS spectra comparison; level 2) for compounds showing i) the loss of -dR in both MS and MS/MS, ii) product ions in MS/MS deriving from the DNA adduct modification, iii) product ions in MS/MS typical of one of the 5 nucleobases; level 3) was assigned to compounds showing the typical fragmentation pattern of DNA adducts, i.e. the loss of $\mathrm{dR}$ in both MS and MS/MS, but with no informative fragments in MS/MS related to the DNA adduct modification and to the nucleobase.

\section{- RESULTS AND DISCUSSION}

Early studies on DNA damage mainly utilized ${ }^{32} \mathrm{P}-$ postlabeling methods to measure adduct levels. However, in the past years, UHPLC-HRMS became the gold standard for DNA adduct analysis, especially due to the possibility of providing a reliable identification of DNA adducts and performing untargeted DNA adductomics ${ }^{4}$. Despite the high potentiality of such a powerful technique, method development is still a frontier, especially for improving the instrumental sensitivity. Although measurements of up to 1 DNA adduct in $10^{10}$ unmodified nucleotides have been accomplished in targeted methods ${ }^{18}$, untargeted methods do not reach similar levels of sensitivity. ${ }^{10}$ Therefore, several chromatographic and mass spectrometric parameters were optimized for developing a sensitive untargeted method. The formation of several adducts such as $[\mathrm{M}+\mathrm{H}]^{+}$, $[\mathrm{M}+\mathrm{Na}]^{+},[\mathrm{M}+\mathrm{K}]^{+},[2 \mathrm{M}+\mathrm{H}]^{+},[2 \mathrm{M}+\mathrm{Na}]^{+}$, and high levels of the in source fragment $[\mathrm{M}-\mathrm{dR}+\mathrm{H}]^{+}$, was detected from 25 DNA adducts. Therefore, the target of this study was improving the response of the DNA adducts by increasing the $[\mathrm{M}+\mathrm{H}]^{+}$, simultaneously decreasing the other adducts and $[\mathrm{M}-\mathrm{dR}+\mathrm{H}]^{+}$.

\section{Optimization of Chromatographic Conditions}

The DNA adduct response was compared under different chromatographic conditions. Several mobile phases have been used in the past. These include water $\left(\mathrm{H}_{2} \mathrm{O}\right)$ as mobile phase $\mathrm{A}$ and methanol $(\mathrm{MeOH})$ or acetonitrile $(\mathrm{ACN})$ as mobile phase $\mathrm{B}$, both phases either with or without the addition of acetic acid $\left(\mathrm{CH}_{3} \mathrm{COOH}\right)$, formic acid $(\mathrm{HCOOH})$, or ammonium acetate $\left(\mathrm{CH}_{3} \mathrm{COONH}_{4}\right) \cdot{ }^{10,19-21}$ However, to the best of our knowledge, there is no study showing a comprehensive comparison of the commonly used chromatographic conditions for the analysis of different classes of DNA adducts. Only one study reported the comparison of ammonium acetate, formate $\left(\mathrm{HCOONH}_{4}\right)$ and bicarbonate $\left(\mathrm{NH}_{4} \mathrm{HCO}_{3}\right)$ for the analysis of acetaldehyde DNA adducts, showing the last as the most promising additive. ${ }^{22}$ This was also confirmed in a further study where HILIC chromatography was used for detecting acrolein DNA adducts. ${ }^{23}$ Based on this, chromatographic conditions using different concentrations of $\mathrm{HCOOH}, \mathrm{CH}_{3} \mathrm{COOH}$, $\mathrm{CH}_{3} \mathrm{COONH}_{4}, \mathrm{HCOONH}_{4}$ and $\mathrm{NH}_{4} \mathrm{HCO}_{3}$ in $\mathrm{H}_{2} \mathrm{O}$ as mobile phase $\mathrm{A}$ and $\mathrm{MeOH}$ as mobile phase $\mathrm{B}$ were tested (Table $\mathrm{S} 1$ ). ACN as phase B was excluded since $\mathrm{MeOH}$ showed much higher ionization efficiency for all the DNA adducts in preliminary experiments.

Each different additive was evaluated at increasing concentrations on the same day. The best concentration of each additive was chosen and compared with the others on the same day to avoid instrumental variability. The mobile phase evaluation was carried out only in positive polarity mode since most of the DNA adducts show better ionization in positive mode. The area of the DNA adduct peaks, the ratio between $[\mathrm{M}+\mathrm{H}]^{+}$and $[\mathrm{M}-\mathrm{dR}+\mathrm{H}]^{+}$and the ratio between $[\mathrm{M}+\mathrm{H}]^{+}$ and other adducts was used for comparison as shown in Section 4 of the Supporting Information. Only the 4 most abundant adducts have been reported for each condition. Although the intensities of the $[\mathrm{M}+\mathrm{H}]^{+}$and $[\mathrm{M}-\mathrm{dR}+\mathrm{H}]^{+}$are changing by using different mobile 
phases, the ratio between $[\mathrm{M}+\mathrm{H}]^{+}$and $[\mathrm{M}-\mathrm{dR}+\mathrm{H}]^{+}$is almost constant under all the conditions, so this was not the main parameter evaluated for mobile phase selection.

The intensity of the signal of both $[\mathrm{M}+\mathrm{H}]^{+}$and $[\mathrm{M}-$ $\mathrm{dR}+\mathrm{H}]^{+}$decreased and the ratio $[\mathrm{M}+\mathrm{H}]^{+} /[\mathrm{M}+\mathrm{Na}]^{+}$ increased by increasing the concentration of $\mathrm{HCOOH}$ except for a few compounds, where the best concentration of $\mathrm{HCOOH}$ was $0.1 \%$ (Figure S4.1). Therefore, $0.05 \% \mathrm{HCOOH}$ was chosen for further comparison. There is not a particular trend depending on the concentration of $\mathrm{CH}_{3} \mathrm{COOH}$; the highest signal was obtained at $0.3 \%$ (Figure S4.2), which also decreased the formation of $\mathrm{Na}^{+}$adducts, $\mathrm{K}^{+}$adducts and dimers and was therefore chosen as the best condition. The only case in which an increase of the ratio between $[\mathrm{M}+\mathrm{H}]^{+}$and $[\mathrm{M}-\mathrm{dR}+\mathrm{H}]^{+}$is observed by increasing the concentration of the additive is shown for $\mathrm{CH}_{3} \mathrm{COONH}_{4}$ (Figure S4.3). Since the area of $[\mathrm{M}+\mathrm{H}]^{+}$ increases by increasing the concentration of the additive for all the compounds but thymine, Me-dC, and M1$\mathrm{dG}, 0.5 \mathrm{mM} \mathrm{CH}_{3} \mathrm{COONH}_{4}$ was chosen as the best condition. An interesting behavior was observed for $\mathrm{CH}_{3} \mathrm{COONH}_{4}$, where the formation of ions with a neutral mass difference of 58.053, corresponding to
$\left[\mathrm{M}+\mathrm{C}_{2} \mathrm{H}_{6} \mathrm{~N}_{2}+\mathrm{H}\right]^{+}$, were detected for some of the compounds. In a previous paper, ${ }^{24}$ the authors suggested that an ion at $[\mathrm{M}+59]^{+}$is due to a contaminant in the $\mathrm{CH}_{3} \mathrm{COONH}_{4}$, most likely acetamidine. In the case of $\mathrm{HCOONH}_{4}$, some of the compounds show a slight signal decrease with increasing concentration of the additive (Figure S4.4). However, most of the compounds show increased signals, reaching a maximum at $5 \mathrm{mM} \mathrm{HCOONH}_{4}$, which was chosen as the best condition for further comparison. Finally, in the case of $\mathrm{NH}_{4} \mathrm{HCO}_{3}$, a very heterogeneous behavior was shown among the compounds (Figure S4.5). Since no particular trend was observed, $10 \mathrm{mM}$ concentration was chosen giving the highest average signal over all the DNA adducts. In conclusion, $0.05 \% \mathrm{HCOOH}, 0.3 \% \mathrm{CH}_{3} \mathrm{COOH}, 5 \mathrm{mM}$ $\mathrm{CH}_{3} \mathrm{COONH}_{4}, \quad 5 \mathrm{mM} \mathrm{HCOONH}_{4}$, and $10 \mathrm{mM}$ $\mathrm{NH}_{4} \mathrm{HCO}_{3}$ were chosen for further analysis in comparison to $\mathrm{H}_{2} \mathrm{O}$ and $\mathrm{MeOH}$ without any additive.

As shown in Figure 1, the best mobile phase was $10 \mathrm{mM}$ $\mathrm{NH}_{4} \mathrm{HCO}_{3}$ for the majority of the DNA adducts, showing an increase of up to 2 orders of magnitude compared to the other conditions and a general decrease of $\mathrm{Na}^{+}$adducts. Only few adducts, i.e. uridine, $\mathrm{dU}$, and oxo-dG, show a better response when

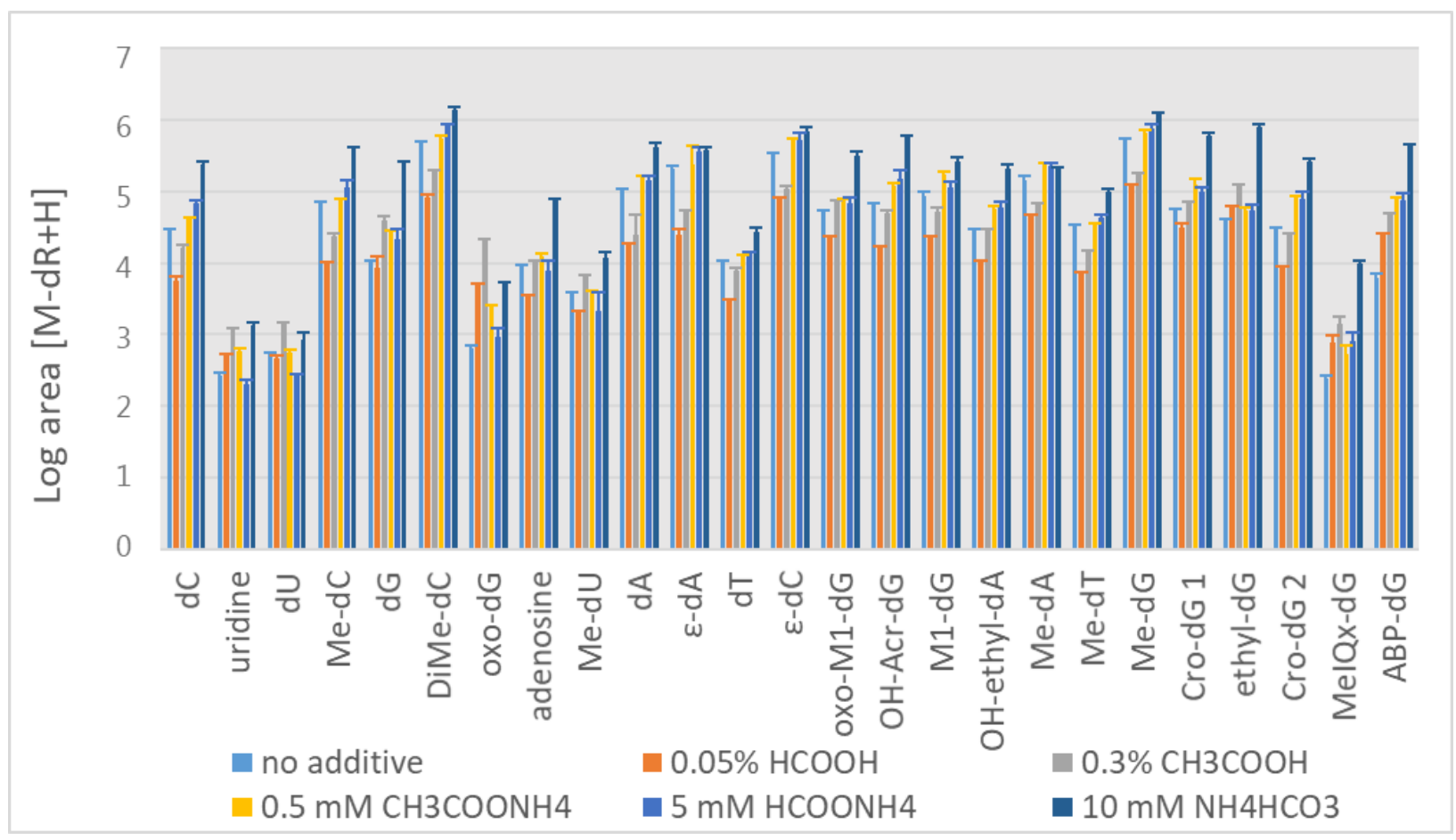

Figure1: $\log$ of the area of the $[\mathrm{M}-\mathrm{dR}+\mathrm{H}]^{+}$ion of the DNA adduct standards analyzed by using the following additives in the mobile phases: no additive, $0.05 \% \mathrm{HCOOH}, 0.3 \% \mathrm{CH}_{3} \mathrm{COOH}, 5 \mathrm{mM} \mathrm{CH} \mathrm{COONH}_{4}, 5 \mathrm{mM}^{2}$ $\mathrm{HCOONH}_{4}$ and $10 \mathrm{mM} \mathrm{NH} \mathrm{HCO}_{3}$. 
$\mathrm{CH}_{3} \mathrm{COOH}$ is used. In addition, the mentioned compounds together with Me-dU, dT, and Me-dT, show very little $[\mathrm{M}+\mathrm{H}]^{+}$and only the $[\mathrm{M}+\mathrm{Na}]^{+}$under almost all the conditions. This is explained by the acidity and poor proton affinity of uracil and thymine ${ }^{25}$ which could be improved by the use of more acidic conditions. Uracil and thymine derived adducts also show a better response in negative polarity (data not shown), suggesting the need for a separate acquisition and a further optimization in future works. The chromatographic parameters were also taken into consideration in the choice of the mobile phases. However, there was no major changes in the chromatographic performance under the different conditions, all showing optimal retention, peak shape, resolution and reproducibility (Figures S4.6, S4.7, S4.8, Table S4.1).

In order to further reduce the formation of $[\mathrm{M}+\mathrm{Na}]^{+}$or $[\mathrm{M}+\mathrm{K}]^{+}$, a final comparison was performed by using different mobile phase bottles, i.e. common glass bottles vs low density polyethylene LDPE bottles (Waters). A slight reduction of $[\mathrm{M}+\mathrm{Na}]^{+}$and a clear reduction of $[\mathrm{M}+\mathrm{K}]^{+}$is shown for most of the adducts when using the LDPE bottles. However, this did not improve the sensitivity of the method, since the intensity of the $[\mathrm{M}+\mathrm{H}]^{+}$remained constant (Figure S4.9).

\section{Optimization of Mass spectrometric Conditions}

Once the best chromatographic condition was chosen, a comprehensive mass spectrometric optimization was carried out with the purpose of both increasing the intensity of the signal and reducing the loss of - $\mathrm{dR}$ from the DNA adducts. The parameters investigated concerned different parts of the instrumentation included ESI related parameters (capillary and sampling cone voltage, source temperature, desolvation gas flow and temperature), collision energies (a minimum collision energy is always required in qTOF instruments for assuring the transmission of the ions), acquisition mode related parameters (mass range, the use of automatic or manual profile mode, which consists in setting the mass range parameters for the quadrupole manually rather than allowing the instrument to automatically optimize it), and detector parameters (detector voltage). The results of the mass spectrometric optimization are shown in terms of both the intensity of the most abundant ion for each DNA adducts, and the ratio between that ion and the DNA adduct after the loss of $-\mathrm{dR}$ (Figure S 5.1). For the capillary and sampling cone there is a remarkable decrease of the signal and increase of the loss of $-\mathrm{dR}$, by increasing the voltages. Therefore, the minimum value was chosen for the capillary voltage and a value of $20 \mathrm{eV}$ was chosen for the cone voltage. An increase in the signal was reported by increasing the desolvation gas temperature. By looking at the acquisition mode related parameters, the mass range did not affect the signal much, and the automatic profile was better than the manual. No particular improvement was shown by changing the source temperature, the desolvation gas flow and the collision energy. The

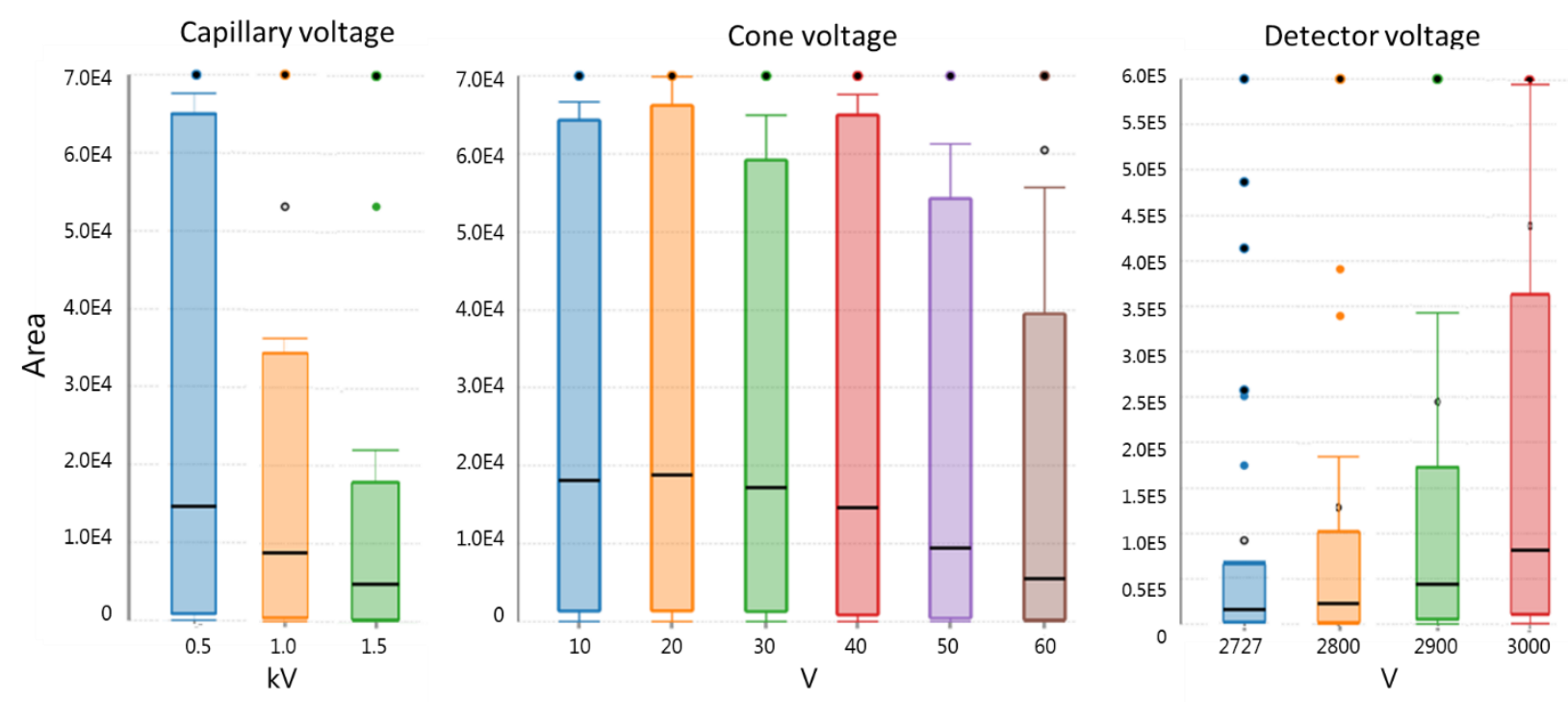

Figure 2: Box-and-Whisker plots showing the distribution of the peak area of the DNA adduct standards at different values of capillary, sampling cone and detector voltage (Graph created with www.goodcalculators.com). 
signal was strongly affected by the detector voltage, which was optimal at $3000 \mathrm{~V}$. This increased the signal up to 10 times; however, when choosing high detector voltages, it is necessary to agree with the Vendor specialists on which is the highest acceptable limit, or the detector could deteriorate. In conclusion, several parameters, especially the capillary voltage, sampling cone voltage and detector voltage caused a remarkable increase of the signal, and 1 or 2 order of magnitudes (Figure 2), was achieved after optimization, while a partial reduction of $-\mathrm{dR}$ loss was achieved for the first two parameters.

\section{Reduction of adsorption processes}

Many other factors, besides the ionization efficiency in the source, can affect the sensitivity of the method. For compounds present in traces such as DNA adducts, adsorption processes can occur on several surfaces, such as the injection vials, the metal surfaces of the chromatographic system and the column. During the optimization process several precautions were taken into consideration for controlling adsorption processes. However, the results often showed a discrete change in the intensity over continuous injection series. In order to explore this issue, 5 different injection vials and 2 different columns were evaluated. From a previous evaluation (data not shown) several vials have been tested and selected for further comparison. In particular, polypropylene vials were excluded, showing a clear decrease in the signal compared to the others. Four different low-adsorptive injection vials were compared with a standard glass vial (LC-MS certified), which resulted as the one showing the highest instability with a clear signal decrease after the first hour for several of the DNA adducts (Figure S6.1). The RSA-Pro vial, is the one showing the highest signal for most of the compounds, but the signal tends to increase after the first hour and then decrease after 4 hours of injections. The other three vials showed a quite stable signal over time, generally up to $16 \mathrm{hrs}$. However, the Quan Recovery vial showed a much lower signal compared to the other vials for M1-dG and Me-dG, whereas the TruView vial showed a lower signal for $\mathrm{ABP}-\mathrm{dG}$ and no signal for MeIQx-dG, suggesting that it is not suitable for the analysis of bulky DNA adducts. In conclusion, the vial showing an acceptable behavior in terms of both intensity and stability for DNA-adduct analysis is the Low Adsorption vial.

The chromatographic column chosen for developing this method, i.e HSST3, has recently been marketed as
Premier HSST3, where a hybrid organic-inorganic surface, based on an ethylene-bridged siloxane chemistry has been applied on the metal surfaces. ${ }^{26}$ This technology resulted in higher intensities for nucleotides, probably because of a reduction of the adsorption of these analytes on the metal oxide layer, which at acid $\mathrm{pH}$ is positively charged. The HSST3 and Premier HSST3 were tested using the DNA adduct standard mix at both $1 \mathrm{ng} \mathrm{mL}^{-1}$ and $10 \mathrm{ng} \mathrm{mL}^{-1}$. Some of the compounds showed a slight decrease in the signal when using the Premier HSST3, however, other compounds showed a significant signal increase (Figure S6.2). As expected, based on the surface chemistry of Premier HSST3, especially the most acidic compounds, i.e. uridine, dU, Me-dU, oxo-dG showed a remarkable improvement. Also the bulky MeIqX-dG showed good results. Further improvement could likely be achieved by using low adsorption HPLC systems available in the market.

\section{Acquisition mode and identification approach}

Several approaches have been employed in the past for the screening of the DNA adducts, often by monitoring the loss of $-\mathrm{dR}$ and the unmodified nucleobase fragment ions. Early DNA adductomics primarily utilized triple quadrupole instrumentation to perform neutral loss screening, whereas more recent studies have taken advantage of $\mathrm{HRMS}^{4}$, which allow to perform different types of acquisition modes such as data dependent acquisition (DDA), ${ }^{27}$ data independent acquisition (DIA), ${ }^{28,29}$ and others. ${ }^{8,19,20}$ Whereas DDA selects specific precursor ions for fragmentation resulting in clean MS/MS spectra, DIA (or $\mathrm{MS}^{\mathrm{E}}$ ) implies the fragmentation of the entire range of ions, requiring elaborated data analysis software for the investigation of the spectra, and also a supplementary MS/MS targeted acquisition for confirming the identity of the compound. However, the selective approach of DDA implies the risk of losing the fragmentation of the least abundant compounds. ${ }^{4}$ This is important for DNA adducts, which are normally present in traces in complex biological matrices rich of highly abundant contaminants. Therefore, $\mathrm{MS}^{\mathrm{E}}$ acquisition at 6 and 20$50 \mathrm{eV}$ for the low and high energy trace, respectively, was chosen to allow detection and fragmentation of a high number of features. The first step of screening consisted in the monitoring of the loss of - $\mathrm{dR}$ across all RTs in high energy trace to find potential DNA adducts. The second step of screening consisted in the monitoring of the loss of the $-\mathrm{dR}$ in the low energy trace 
and the presence of the fragment ions belonging to the nucleobases in high energy trace. Indeed, all the DNA adduct standards showed some in-source fragmentation and this allowed not only introducing an extra level of screening for the DNA adducts, but also to perform a pseudo $\mathrm{MS}^{3}$ fragmentation where the modified nucleobase could be further fragmented in the high energy collision trace, allowing to monitor the fragments related to the unmodified nucleobase within the same experiment. Since not all the DNA adducts show in source fragmentation leading to liberation of the nucleobase ion, features showing the loss of $-\mathrm{dR}$ in high energy trace and either loss $-\mathrm{dR}$ in low energy trace or typical nucleobases fragment ions were selected for further validation. The selected masses were further fragmented through targeted MS/MS (Third step of screening). A summary on the selected features and tentatively identified DNA adducts is provided in the Supporting Information (Table S7.1).

\section{Identification in DNA from Calf thymus and cat colon tissue}

The optimized untargeted method was applied for the identification of DNA adducts from calf thymus and from cat colon, leading to the tentative identification of 13 possible DNA adducts as shown in Table S7.2 and Figure S7.1.

C8-oxo-dG, dU and Me-dC were compared with commercial reference standards and thereby identified at level I as present in commercial calf thymus DNA as well as in freshly isolated cat colon DNA. C8-oxo-dG is one of the most studied DNA adducts and it derives from the oxidative damage to DNA by reactive oxygen species (ROS), which occurs endogenously as part of normal metabolism. ${ }^{31} \mathrm{dU}$ is a result of deamination of cytosine to uracil, which occurs spontaneously by several mechanisms such as simple hydrolysis, attack by nitric oxide-derived species or ROS during inflammation, and by the activity of deaminase enzymes. ${ }^{32,33} \mathrm{Me}-\mathrm{dC}$ is a result of usual epigenetic modification processes. ${ }^{34}$ High abundance of these adducts is therefore expected.

Two isomers of carboxy-Me-dG and two isomers of carboxyethyl-dG were fragmented through targeted MS/MS and tentatively identified at level II in DNA from calf thymus as well as cat colon. The two isomers are probably due to different positions of the carboxymethyl and carboxyethyl group on the $\mathrm{dG}$. However, the identities are difficult to confirm since peaks are partially overlapping, showing the same fragmentation pattern. Carboxy-Me-dG and carboxyethyl-dG have the same accurate mass as the adducts from common glycotoxins, glyoxal-dG and Me-glyoxal-dG, respectively. However, in both cases and in both isomers, the fragmentation pattern strongly suggests the identities reported here, due to the loss of $\mathrm{CO}_{2}$. Glyoxal-dG has been found to be unstable and is partially transformed to carboxy-Me-dG. ${ }^{35}$ The compounds may therefore derive mainly from rearrangements of adducts formed by known glycotoxins. In previous works, O6-carboxy-Me-dG have been found in colon tissues ${ }^{9}$ and exfoliated colonocytes. ${ }^{36} \mathrm{Me}$-glyoxal is a common byproduct of the ubiquitous glycolysis pathway and reacts with $\mathrm{dG}$ to form $\mathrm{N}^{2}$-carboxyethyl-dG. ${ }^{37}$

Deoxyxanthosine and deoxyinosine were also identified at level II in both DNA from calf thymus and cat experiments. These two adducts are a result of $\mathrm{dG}$ and dA deamination in DNA, respectively, as in the case of $\mathrm{dU} .^{32,33}$

Finally, four unknown adducts were detected in DNA from calf thymus, two of them conjugated with cytosine. However, since the fragmentation pattern was not informative enough they have been assigned at level III.

In a previous work, ${ }^{9}$ where an untargeted DNA adductomics approach was used in colon tissues, 17 DNA adducts were identified through HRMS, including O6-Carboxy-Me-dG and other adducts not found in the current work. However, the method implied the analysis of nucleobases obtained after acidic hydrolysis rather than nucleosides. In other works on other samples such as lung tissues, ${ }^{10}$ and cells, ${ }^{20}$ a range of 50-150 DNA adducts were identified. Here different sample preparation and instrumental methods, including nano-ESI-HPLC and fractionation, were used for the analysis. The method developed in this work represents a promising step for untargeted DNA adductomics. Further sensitivity of the analytical method and identification of additional adducts will further enhance this technology for translation into cancer research.

\section{- CONCLUSION}

In this work, a sensitive UHPLC-HRMS untargeted method coupled with a DNA adduct database was developed for the detection and identification of DNA adducts. The optimization of the chromatographic conditions showed the mobile phases containing $10 \mathrm{mM}$ ammonium bicarbonate to be the best condition in 
terms of signal enhancement of a mix of DNA adducts, used as reference standards. The capillary voltage, the sampling cone voltage and the detector voltage highly affected the response of the DNA adducts. Also, a low adsorption vial was selected for the highest stability of the signal over time. Finally, a hybrid surface coated HSST3 premier column was showing a minor adsorption of the DNA adducts and therefore preferred to an ordinary HSST3 column. These optimised analytical conditions were chosen for the analysis of DNA from calf thymus and cat colon using $\mathrm{MS}^{\mathrm{E}}$ acquisition, where the DNA adducts were screened by monitoring the loss of -dR both in-source and in the fragmentation spectra, allowing further fragmentation of the ions belonging to the unmodified nucleobases. The method led to the identification of 13 DNA adducts in DNA from calf thymus and cat colon, showing good promise for the application of this untargeted method in future human studies. Further improvement of the method should be addressed in future works especially regarding the optimization of the sample preparation procedures, in order to improve the recovery of DNA adducts and decrease matrix effects.

\section{Acknowledgments}

This work and Giorgia La Barbera were founded by the European Commision for the H2020 Marie Curie Slodowska Individual Fellowships (grant $n^{\circ}$ 843892). Lars Ove Dragsted was supported by a Semper Ardens grant from the Carlsberg Foundation. Prof Charlotte Reinhard Bjørnvad, provided the cat colon sample.

\section{- REFERENCES}

(1) Balbo, S.; Turesky, R. J.; Villalta, P. W. DNA Adductomics. Chem. Res. Toxicol. 2014, 27 (3), 356366.

(2) Loeb, L. A.; Harris, C. C. Advances in Chemical Carcinogenesis: A Historical Review and Prospective. Cancer Res. 2008, 68 (17), 6863.

(3) Tretyakova, N.; Villalta, P. W.; Kotapati, S. Mass Spectrometry of Structurally Modified DNA. Chem. Rev. 2013, 113 (4), 2395-2436.

(4) Villalta, P.; Balbo, S. The Future of DNA Adductomic Analysis. Int. J. Mol. Sci. 2017, 18 (9), 1870.

(5) Murray, K. J.; Carlson, E. S.; Stornetta, A.; Balskus, E. P.; Villalta, P. W.; Balbo, S. Extension of Diagnostic Fragmentation Filtering for Automated Discovery in DNA Adductomics. Anal. Chem. 2021, 93 (14), 57545762.

(6) Sousa, P. F. M.; Martella, G.; Åberg, K. M.; Esfahani, B.;
Motwani, H. V. NLossFinder-A Graphical User Interface Program for the Nontargeted Detection of DNA Adducts. Toxics 2021, Vol. 9, Page 78 2021, 9 (4), 78.

(7) Hemeryck, L. Y.; Vanhaecke, L. Diet-Related DNA Adduct Formation in Relation to Carcinogenesis. Nutr. Rev. 2016, 74 (8), 475-489.

(8) Guo, J.; Villalta, P. W.; Turesky, R. J. Data-Independent Mass Spectrometry Approach for Screening and Identification of DNA Adducts. Anal. Chem. 2017, 89 (21), 11728-11736.

(9) Hemeryck, L. Y.; Decloedt, A. I.; Vanden Bussche, J.; Geboes, K. P.; Vanhaecke, L. High Resolution Mass Spectrometry Based Profiling of Diet-Related Deoxyribonucleic Acid Adducts. Anal. Chim. Acta 2015, 892, 123-131.

(10) Carrà, A.; Guidolin, V.; Dator, R. P.; Upadhyaya, P.; Kassie, F.; Villalta, P. W.; Balbo, S. Targeted High Resolution LC/MS3 Adductomics Method for the Characterization of Endogenous DNA Damage. Front. Chem. 2019, 7, 658.

(11) Tudek, B.; Speina, E. Oxidatively Damaged DNA and Its Repair in Colon Carcinogenesis. Mutat. Res. Mol. Mech. Mutagen. 2012, 736 (1-2), 82-92.

(12) Murphy, N.; Moreno, V.; Hughes, D. J.; Vodicka, L.; Vodicka, P.; Aglago, E. K.; Gunter, M. J.; Jenab, M. Lifestyle and Dietary Environmental Factors in Colorectal Cancer Susceptibility. Mol. Aspects Med. 2019, 69, 2-9.

(13) Chambers, M. C.; MacLean, B.; Burke, R.; Amodei, D.; Ruderman, D. L.; Neumann, S.; Gatto, L.; Fischer, B.; Pratt, B.; Egertson, J.; Hoff, K.; Kessner, D.; Tasman, N.; Shulman, N.; Frewen, B.; Baker, T. A.; Brusniak, M. Y.; Paulse, C.; Creasy, D.; Flashner, L.; Kani, K.; Moulding, C.; Seymour, S. L.; Nuwaysir, L. M.; Lefebvre, B.; Kuhlmann, F.; Roark, J.; Rainer, P.; Detlev, S.; Hemenway, T.; Huhmer, A.; Langridge, J.; Connolly, B.; Chadick, T.; Holly, K.; Eckels, J.; Deutsch, E. W.; Moritz, R. L.; Katz, J. E.; Agus, D. B.; MacCoss, M.; Tabb, D. L.; Mallick, P. A CrossPlatform Toolkit for Mass Spectrometry and Proteomics. Nature Biotechnology. Nature Publishing Group October 10, 2012, pp 918-920.

(14) Pluskal, T.; Castillo, S.; Villar-Briones, A.; Orešič, M. MZmine 2: Modular Framework for Processing, Visualizing, and Analyzing Mass Spectrometry-Based Molecular Profile Data. BMC Bioinformatics 2010, 11.

(15) La Barbera, G.; Antonelli, M.; Cavaliere, C.; Cruciani, G.; Goracci, L.; Montone, C. M.; Piovesana, S.; Laganà, A.; Capriotti, A. L. Delving into the Polar Lipidome by Optimized Chromatographic Separation, High-Resolution Mass Spectrometry, and Comprehensive Identification with Lipostar: Microalgae as Case Study. Anal. Chem. 2018, 90 (20), 12230-12238.

(16) Schymanski, E. L.; Jeon, J.; Gulde, R.; Fenner, K.; Ruff, 
M.; Singer, H. P.; Hollender, J. Identifying Small Molecules via High Resolution Mass Spectrometry: Communicating Confidence. Environ. Sci. Technol. 2014, 48 (4), 2097-2098.

(17) Wilson, M. R.; Jiang, Y.; Villalta, P. W.; Stornetta, A.; Boudreau, P. D.; Carrá, A.; Brennan, C. A.; Chun, E.; Ngo, L.; Samson, L. D.; Engelward, B. P.; Garrett, W. S.; Balbo, S.; Balskus, E. P. The Human Gut Bacterial Genotoxin Colibactin Alkylates DNA. Science (80-. ). 2019, 363 (6428).

(18) Villalta, P. W.; Hochalter, J. B.; Hecht, S. S. Ultrasensitive High-Resolution Mass Spectrometric Analysis of a DNA Adduct of the Carcinogen Benzo[a]Pyrene in Human Lung. Anal. Chem. 2017, 89 (23), 12735-12742.

(19) Balbo, S.; Hecht, S. S.; Upadhyaya, P.; Villalta, P. W. Application of a High-Resolution Mass-SpectrometryBased DNA Adductomics Approach for Identification of DNA Adducts in Complex Mixtures. Anal. Chem. 2014, $\quad 86 \quad$ (3), $1744-1752$. https://doi.org/10.1021/AC403565M.

(20) Stornetta, A.; Villalta, P. W.; Hecht, S. S.; Sturla, S. J.; Balbo, S. Screening for DNA Alkylation Mono and Cross-Linked Adducts with a Comprehensive LC-MS3 Adductomic Approach. Anal. Chem. 2015, 87 (23), 11706-11713.

(21) LY, H.; C, R.; E, D. P.; L, V. DNA Adduct Profiling of in Vitro Colonic Meat Digests to Map Red vs. White Meat Genotoxicity. Food Chem. Toxicol. 2018, 115, 73-87.

(22) Yin, R.; Liu, S.; Zhao, C.; Lu, M.; Tang, M.-S.; Wang, H. An Ammonium Bicarbonate-Enhanced Stable Isotope Dilution UHPLC-MS/MS Method for Sensitive and Accurate Quantification of Acrolein-DNA Adducts in Human Leukocytes. 2013.

(23) Murakami, H.; Horiba, R.; Iwata, T.; Miki, Y.; Uno, B.; Sakai, T.; Kaneko, K.; Ishihama, Y.; Teshima, N.; Esaka, Y. Progress in a Selective Method for the Determination of the Acetaldehyde-Derived DNA Adducts by Using HILIC-ESI-MS/MS. Talanta 2018, $177,12-17$.

(24) Abián, J.; Sáchez-Baeza, F.; Gelpí, E.; Barceló, D. On the Origin of Some Controversial Ions ( $\mathrm{m} / \mathrm{z} 59,60,77$, and 119) in the Thermospray Reagent Plasma from Ammonium Acetate. J. Am. Soc. Mass Spectrom. 1994, 5 (3), 186-193.

(25) Liguori, A.; Napoli, A.; Sindona, G. Survey of the Proton Affinities of Adenine, Cytosine, Thymine and Uracil Dideoxyribonucleosides, Deoxyribonucleosides and Ribonucleosides †. J. MASS Spectrom. J. Mass Spectrom 2000, 35, 139-144.

(26) DeLano, M.; Walter, T. H.; Lauber, M. A.; Gilar, M.; Jung, M. C.; Nguyen, J. M.; Boissel, C.; Patel, A. V.; Bates-Harrison, A.; Wyndham, K. D. Using Hybrid Organic-Inorganic Surface Technology to Mitigate
Analyte Interactions with Metal Surfaces in UHPLC. Anal. Chem. 2021, 93 (14), 5773-5781.

(27) Van den Driessche, B.; Van Dongen, W.; Lemière, F.; Esmans, E. L. Implementation of Data-Dependent Acquisitions in the Study of Melphalan DNA Adducts by Miniaturized Liquid Chromatography Coupled to Electrospray Tandem Mass Spectrometry. Rapid Commun. Mass Spectrom. 2004, 18 (17), 2001-2007.

(28) Ishino, K.; Kato, T.; Kato, M.; Shibata, T.; Watanabe, M.; Wakabayashi, K.; Nakagama, H.; Totsuka, Y. Comprehensive DNA Adduct Analysis Reveals Pulmonary Inflammatory Response Contributes to Genotoxic Action of Magnetite Nanoparticles. Int. J. Mol. Sci. 2015, 16 (2), 3474-3492.

(29) Schmied-Tobies, M. I. H.; Paschke, H.; Reemtsma, T. Combined Chemoassay and Mass Spectrometric Approach to Study the Reactive Potential of Electrophiles towards Deoxynucleosides as Model for DNA. Chemosphere 2016, 151, 263-270.

(30) Guo, J.; Turesky, R. J.; Tarifa, A.; DeCaprio, A. P.; Cooke, M. S.; Walmsley, S. J.; Villalta, P. W. Development of a DNA Adductome Mass Spectral Database. Chem. Res. Toxicol. 2020, 33 (4), 852-854.

(31) Dizdaroglu, M.; Jaruga, P. Mechanisms of Free RadicalInduced Damage to DNA. 2012, 46 (4), 382-419.

(32) Pang, B.; McFaline, J. L.; Burgis, N. E.; Dong, M.; Taghizadeh, K.; Sullivan, M. R.; Elmquist, C. E.; Cunningham, R. P.; Dedon, P. C. Defects in Purine Nucleotide Metabolism Lead to Substantial Incorporation of Xanthine and Hypoxanthine into DNA and RNA. Proc. Natl. Acad. Sci. 2012, 109 (7), 23192324.

(33) Kow, Y. W. Repair of Deaminated Bases in DNA. Free Radic. Biol. Med. 2002, 33 (7), 886-893.

(34) Kumar, S.; Chinnusamy, V.; Mohapatra, T. Epigenetics of Modified DNA Bases: 5-Methylcytosine and Beyond. Front. Genet. 2018, 9.

(35) Wang, H.; Cao, H.; Wang, Y. Quantification of N2Carboxymethyl-2'-Deoxyguanosine in Calf-Thymus DNA and Cultured Human Kidney Epithelial Cells by Capillary HPLC-Tandem Mass Spectrometry Coupled with Stable Isotope-Dilution Method. Chem. Res. Toxicol. 2010, 23 (1), 74.

(36) MH, L.; N, B.; T, B.; R, B.; AJ, C.; J, P.; DE, S.; SA, B. Red Meat Enhances the Colonic Formation of the DNA Adduct O6-Carboxymethyl Guanine: Implications for Colorectal Cancer Risk. Cancer Res. 2006, 66 (3), 1859-1865.

(37) Matthias Frischmann, †; Clemens Bidmon, †; Jürgen Angerer, $\ddagger$ and; Monika Pischetsrieder*, $\dagger$. Identification of DNA Adducts of Methylglyoxal. Chem. Res. Toxicol. 2005, 18 (10), 1586-1592. 